\title{
Los valores vinculados a la competencia del trabajo en equipo en entornos virtuales de aprendizaje. Un estudio en la Universidad Técnica Particular de Loja (Ecuador)
}

\section{Values linked to generic competence of teamwork in virtual learning environments. A study at the Universidad Técnica Particular de Loja (Ecuador)}

\author{
Marta RUÍZ-CORBELLA y Rosario de RIVAS MANZANO \\ Universidad Nacional de Educación a Distancia \\ y Universidad Técnica Particular de Loja (Ecuador)
}

Recibido: Junio 2014

Aceptado: Septiembre 2014

\section{Resumen}

Un componente clave de toda competencia genérica es el valor, cuestión mencionada, pero escasamente atendida en el proceso formativo y, menos aún, evaluada. Encontramos estudios y experiencias que abordan la formación en destrezas, conocimientos, etc., pero no es tan habitual las dirigidas a su dimensión axiológica. Por ello es necesario identificar los valores implícitos en estas, para poder incorporarlas en la dinámica formativa en cada asignatura y en su evaluación.

Los objetivos de este trabajo se apoyan en un estudio de carácter descriptivo centrado en un caso único con recogida de datos a partir de un cuestionario dirigido a los actores del proceso de enseñanza a distancia en un entorno virtual de aprendizaje. Para ello se diseñaron, validaron y aplicaron dos cuestionarios, uno dirigido a profesores y otro a estudiantes, con preguntas que permitieran evaluar cómo los primeros asumen y transmiten estos valores y cómo los perciben los segundos.

Los resultados obtenidos permiten concluir: 1) Hay una marcada diferencia entre el sentir de los profesores en la asunción y transmisión de estos valores y la percepción de los estudiantes. 2) Los profesores, aunque reconocen falencias en algunos aspectos de la formación axiológica de sus estudiantes, no son suficientemente conscientes de estas diferencias significativas. 3) Es necesario que la institución, identificadas las debilidades de los profesores, establezca líneas de trabajo y nuevas acciones que ayuden a alcanzar los objetivos de una formación de calidad.

Este trabajo representa una primera aproximación a un tema que, por su importancia, necesita ser profundizado y continuado a lo largo del tiempo. Gracias a los cuestionarios diseñados y validados podemos contrastar estos resultados en otros entornos virtuales de aprendizaje, para analizar qué formación se está favoreciendo en nuestras instituciones universitarias. Identificar esta realidad es la vía para poder presentar planes de mejora en las titulaciones que se imparten.

Palabas clave: educación superior, educación a distancia, educación virtual, competencias genéricas, formación en valores, trabajo en equipo. 


\begin{abstract}
An important component of any generic competence is the value. This matter is mentioned but scarcely attended in the teaching process and is poorly evaluated. Studies and experiences that address training in skills, knowledge, etc. were found. However, it is not so common to address the axiological dimension of each competence. Therefore, it is necessary to identify the values implicit in any generic competence, in order to incorporate this to the dynamic training in each subject, and assess acquisition.

The objectives of this work are based on a descriptive study focused on a single case with data collecting from a survey completed by participants in the process of virtual distance education. To this end, we designed, validated and applied two questionnaires, one for teachers and another one for students. The surveys have questions that have allowed the assessment on how these values are assumed and transmitted by teachers and also on how the students perceive them.

The results indicate that: 1) There is a strong difference between the feelings of the teachers about the assumption and transmission of these values, and the perception of the students. 2) Teachers even though recognizing the shortcomings in some aspects of the axiological formation of their students, are not sufficiently aware of these important differences. 3) When the institution has identified the weaknesses of the teachers it is necessary to set lines of work to aid them in the process of pondering the situation and to propound new actions that help with the achievement of the objectives of a quality education.

This paper represents a first approach to this subject, which because of its importance, further deepening will be necessary. Thanks to the designed and validated questionnaires we can compare these results in other virtual learning environments, to analyze what is being promoted in our universities. Identify this reality is the way to be able to submit improvement plans in the degrees offered.
\end{abstract}

Key words: higher education, distance education, virtual classrooms, generic competences, values education, teamwork.

En la literatura científica de esta última década (Clemente y Escribá, 2013; Yañiz y Villardon, 2012; Benhayón, 2011; Villa y Poblete, 2011; Sánchez-Elvira et al, 2010; Pallisera, 2010; etc.) se ha resaltado la relevancia de la formación en competencias genéricas en la educación superior como respuesta al perfil profesional que cada titulación debe afrontar en el marco de las necesidades sociales del entorno en el que está enclavado. A la vez que desarrolla las capacidades necesarias para el empleo en la comunidad en la que tendrán que ejercer estos profesionales (Beneittone, González y Wagenaar, 2014).

Implícitos en estos objetivos, resaltan estos autores, está el desarrollo personal de cada estudiante, que redundará en el progreso social, íntimamente relacionado con los valores adquiridos y consolidados a lo largo de la formación universitaria. Toda esta realidad conlleva el que deba existir un trabajo coordinado entre la universidad y los diferentes actores sociales. En este sentido, la formación que se oferta en las instituciones universitarias se dirige a consolidar y afianzar no solo competencias específicas propias de una titulación que reflejan su saber profesional y su saber hacer, sino también, y aún 
más valorados por los agentes sociales, las transversales o genéricas ${ }^{1}$ que hacen efectivo el saber estar y ser (Corominas et al, 2006; Corominas, 2001). Precisamente, los conocimientos que se emplean, a lo largo de la vida, en todo contexto personal, social, formativo, laboral, etc. Es decir, la capacidad de combinar “(...) la capacitación y calificación, adquirida para la formación técnica y profesional, con el comportamiento social, la aptitud para el trabajo en equipo, la iniciativa, el gusto por el riesgo, etc.” (Corominas et al, 2006, p.303). Competencias que integran el saber, el saber hacer y el saber ser de los profesionales del mañana. Además, en los diferentes informes y estudios sobre la formación en la educación superior (Beneitone et al., 2007; Beneitone et al. 2014; ANECA, 2007; OECD, 2005; Villa y Poblete, 2008; University of Cambridge, 2008; etc.), son las más destacadas, ya que "facilitan el desarrollo de una verdadera educación integral, puesto que engloban todas las dimensiones del ser humano. Como consecuencia, suponen un referente obligado para superar una enseñanza meramente academicista y orientar nuestra tarea hacia la formación de una ciudadanía crítica y unos profesionales competentes” (Blanco, 2009, p.25).

Un componente clave de toda competencia genérica es el valor, cuestión mencionada, pero escasamente atendida en el proceso formativo y, menos aún, evaluada. Encontramos estudios y experiencias que abordan la formación de diferentes destrezas, habilidades, conocimientos, etc. ${ }^{2}$, sin embargo son escasas las investigaciones o innovaciones dirigidas a la dimensión axiológica de cada competencia, a pesar de ser un eje relevante de todo proceso formativo. No debemos olvidar que "una comunidad universitaria que se propone transmitir e influenciar tiene unas connotaciones éticas nada desdeñables. Sin ir más lejos, se trata de una comunidad que no se conforma con asumir la realidad, sino que pretende transformarla si esta lo requiere; que no se limita a conocer la verdad de las cosas, sino que quiere interpretarla según sean las circunstancias; (...)” (Esteban Bara y Martínez, 2012, p.82).

Al igual que en todo nivel educativo, la universidad demanda una formación axiológica que también debemos atender, como parte de la formación integral de todo egresado.

La formación integral es propia de todo universitario, independiente de la modalidad en que curse sus estudios, máxime cuando el desarrollo tecnológico actual, aplicado a la educación superior, está cambiando radicalmente este escenario educativo. Las tecnologías de la información y la comunicación (TIC), enriquecidas con herramientas interactivas que promueven y facilitan el trabajo colaborativo y permiten una interacción real y personalizada, han permeado el mundo universitario y han desdibujado las barreras entre lo presencial y a distancia permitiendo una mayor

\footnotetext{
${ }^{1}$ No hay unanimidad a la hora de aportar un término aprobado por todos que recoja el contenido de estas competencias. Son conocidas la utilización de competencias clave (key), comunes, centrales (core), habilidades, etc., lo que conlleva cierta confusión en torno a este concepto.

${ }^{2}$ Es ingente la literatura en torno a este tema. Sirvan como ejemplo, los congresos, y sus actas, que periódicamente se celebran en nuestro país: Congreso Internacional sobre el enfoque basado en competencias; Congreso Internacional de Competencias básicas; Congreso Internacional de Docencia Universitaria e Innovación; Jornadas RED-U y un largo etcétera.
} 
interacción sincrónica y asincrónica entre profesor-estudiante y estudiantes entre sí (García Aretio, 2014). La necesidad de formar en competencias genéricas y, en especial, en los valores que las impregnan, se debe aplicar en toda enseñanza universitaria, independientemente del entorno en el que se imparta. No olvidemos que todo proceso educativo tiene que ocuparse y preocuparse por la formación axiológica, ya que en toda educación los valores forman parte del objetivo de esa acción educativa (Duart, 2003, García López et al, 2009), se tenga o no conciencia de ello (Maldonado, 2010). Es necesario que la educación superior opte también por un claro y activo compromiso con los valores, especialmente con los que contribuyen a una cultura de paz (Tünnermann, 2010), reivindicando la responsabilidad ética, social y ambiental de esta formación (Conferencia Regional de Educación Superior, 2008; Ruiz Corbella y Harris, 2012). No hacerlo sería un craso error en la misión que tiene encomendada esta institución. El carácter público o privado de la Universidad no es lo que determina su sentido y misión, sino más bien que se reconozca como espacio de aprendizaje ético, a partir del cual sus egresados podrán ejercer sus profesiones con la firme voluntad de contribuir a la formación de una sociedad inclusiva, digna y justa (Martínez, 2006, 2008; Esteban Bara y Martínez, 2012; Fresan Orozco, 2009).

\section{El trabajo en equipo, competencia genérica altamente demandada en la actualidad}

En este artículo, de entre todas las competencias genéricas, nos centraremos en el trabajo en equipo por varios motivos:

- Es una de las más demandadas por los empleadores, independientemente de la titulación, ya que el desarrollo laboral, en cualquier entorno, exige el trabajo colaborativo y cooperativo de diferentes profesionales, en los que cada uno aporta sus habilidades y conocimientos para el logro de un objetivo común. Esto conlleva una constante interdependencia que exige, a su vez, la capacidad de comunicación con diferentes actores, de compromiso y motivación, autocontrol, trabajo bajo presión, de gestión de conflictos, de planificación de la tarea, sentido ético, etc., lo que nos indica su relevancia. Además de que "los equipos empiezan a ser la principal forma de trabajo, considerada como una unidad, que puede generar más beneficios a las organizaciones que un simple trabajador" (Torrelles et al, 2011, p.330). El saber trabajar con otros implica conocimientos, destrezas, actitudes y valores para la colaboración, la cooperación, el diálogo, la participación, etc. dirigido a la configuración de un equipo eficaz en el que cada miembro se sabe parte relevante y responsable de ese grupo. En suma, una competencia que “(...) supone la disposición personal y la colaboración con otros en la realización de actividades para lograr objetivos comunes, intercambiando informaciones, asumiendo responsabilidades, resolviendo dificultades que se presentan y contribuyendo a la mejora y desarrollo colectivo” (Torrelles et al., 2011, p.340). 
- Es una de las competencias más referenciadas en las diferentes investigaciones sobre competencias genéricas (Sánchez-Elvira et al., 2010; García-Valcárcel et al., 2012; Clemente y Escriba, 2013; Rekalde y Bujan, 2014).

- El trabajo en equipo es resaltado explícitamente en la misión y visión de la universidad en la que se lleva a cabo esta investigación, la Universidad Técnica Particular de Loja (UTPL) de Ecuador, por lo que interesa comprobar cómo se integra en los diseños formativos y se forma en esta competencia, a través de los valores que la conforman.

A partir de este marco, el objetivo de este trabajo es conocer cómo los profesores incorporan y desarrollan la competencia del trabajo en equipo en el proceso instruccional de su asignatura, centrándonos especialmente en los valores implícitos en ella. Formar en valores no implica solo enseñar una serie de destrezas, transmitir una serie de técnicas que facilitan diferentes dinámicas grupales, sino que, de forma especial, significa interiorizar unos valores que condicionan esa acción en grupo y nuestra disposición a colaborar y comprometerse con él (Duart, 2003). Asimismo, evaluar el logro de la misión y visión de una institución resulta imprescindible para identificar la calidad de la formación de esa universidad.

El trabajo en equipo, como competencia, comprende una serie de valores para su concreción y aplicación, como son responsabilidad, solidaridad, tolerancia, honestidad, respeto, esfuerzo, diálogo, disciplina, confianza ${ }_{2}$ humildad (Murillo, 1994, Pichardo, 2000, López Valverde, 2001, Méndez Martínez, 2003, García Aretio, 2009). Además de que en la enseñanza en entornos virtuales

“(...) la incorporación de las plataformas de aprendizaje como recurso instruccional amplía las oportunidades (...) de educar en valores dado que el trabajo colaborativo, la interacción grupal característicos de los ambientes virtuales de aprendizaje demandan una actitud responsable, honesta, solidaria, respetuosa, libre del estudiante para contribuir a concretar exitosamente los objetivos de una lección o curso" (Páez et al., 2008, p.34).

En nuestro estudio, contrastamos el valor institucional de trabajo en equipo especificado en la misión de la UTPL con esta competencia, cotejándola con la propuesta axiológica que sobre esta metodología llevan a cabo cuatro expertos en educación a distancia (Murillo, 1994; Pichardo, 2000; Rubio Gómez, 2000; García Aretio, 2009). El resultado es la identificación de cinco valores claves que la integran: responsabilidad, diálogo, confianza, generosidad y respeto. Valores esenciales en esta metodología para que pueda darse un aprendizaje colaborativo y cooperativo eficaz apoyados en los distintos canales de interacción.

\section{La evaluación en valores, objetivo de esta investigación}

A partir de esta revisión teórica de los valores implícitos en la competencia genérica del trabajo en equipo, nos propusimos verificar la valoración y percepción de docentes y estudiantes en la modalidad a distancia de la UTPL sobre la integración y desarrollo 
de estos valores en las diferentes asignaturas que se imparten, a partir de los siguientes objetivos específicos:

1. Conocer cómo valoran y perciben los docentes de la modalidad a distancia de UTPL la incorporación de estos valores en acciones docentes determinadas.

2. Conocer cómo valoran y perciben los estudiantes de la modalidad a distancia de UTPL la incorporación de estos valores en acciones docentes determinadas.

3. Identificar posibles diferencias significativas en la percepción de estos valores en ambos actores, con el fin de potenciar su integración en el proceso formativo.

La universidad en la que se enmarca la presente investigación tiene 38 años de experiencia en la oferta de titulaciones a distancia e imparte en la actualidad 19 titulaciones en entornos virtuales de aprendizaje (EVA), que cuenta con más de 30.000 estudiantes.

La institución presenta un claro y explícito compromiso con la formación en competencias genéricas reflejado a través de su misión y visión. Ahora bien, como en todo nivel educativo, no basta con expresar la intención, explícita o implícita, de formar en competencias. Si se quiere alcanzar la excelencia académica, se debe evaluar la calidad de los procesos de enseñanza aprendizaje que se generan a través de los escenarios virtuales específicos de esta universidad $\mathrm{y}$, de forma especial, de la incorporación de competencias genéricas y su nivel de logro. A esta institución le interesa conocer en qué medida sus estudiantes son formados en estas competencias, reflejo de los valores institucionales, expresión clara de una forma de entender la sociedad y la persona.

\section{Metodología de la investigación}

Para la consecución de los objetivos de este trabajo se ha diseñado un estudio de carácter descriptivo centrado en un caso único, la UTPL, con recogida de datos a partir de un cuestionario dirigido a los actores que participan en los EVA: profesores y estudiantes.

Un aspecto importante en las investigaciones apoyadas en la recogida de datos por cuestionario tiene que ver con la selección de la muestra. En este caso se trata de una muestra no probabilística intencional, ya que la población sobre la que se ha llevado a cabo este estudio se centra en una única institución, accesible a los investigadores ${ }^{3}$.

\footnotetext{
${ }^{3}$ Todos los datos, tanto del personal docente, como de los estudiantes y el acceso a ambos grupos, han sido facilitados a los investigadores por la institución.
} 


\section{Muestra}

Se ha trabajado con los 410 profesores que imparten docencia en los EVA de la UTPL en el período académico 2012- 2013, de los que, finalmente, se contó con un total de 330 respuestas válidas, lo que supone el $80,5 \%$ del profesorado objeto de este estudio.

En cuanto a los estudiantes, se decidió trabajar únicamente con los últimos ciclos de todas las titulaciones de Grado de la oferta a distancia de esta universidad, al ser los que mejor pueden aportar su percepción en la adquisición de estos valores. Este grupo asciende a 1.892 matriculados en este mismo período académico, de los que respondieron 1.642, el 86,78\%.

En ambos casos la pérdida de respuestas se debe a cuestionarios no válidos o no contestados.

\section{Instrumentos}

La recogida de datos se ha realizado mediante un cuestionario expresamente creado para este estudio, (Hirsch, 2007; Benítez Zavala, 2009; Silas et al., 2007). Nos inclinamos por este instrumento, válido y fiable, recomendable especialmente en los casos en los que se dan sujetos difícilmente accesibles por la distancia o por su dispersión (García Muñoz, 2003). Lógicamente, la elaboración de este instrumento es uno de los factores centrales de este estudio, por lo que pasamos a detallar el proceso recorrido:

1. Se concretaron los indicadores y descriptores de cada uno de los valores institucionales recogidos en la definición de la misión de esta universidad. Cada valor institucional se correlacionó con las competencias genéricas y los valores implícitos en cada una de éstas, gracias a los cuales pudieran ser evaluados y, por lo tanto, medidos por medio de la percepción de profesores y estudiantes.

2. Una vez identificados y definidos estos valores, se especificaron los indicadores que los configuran, apoyándonos en la bibliografía pertinente y en la reflexión sobre los valores que se pretendían evaluar.

3. A partir de la identificación de los valores y sus indicadores, se realizó una matriz que permitió formular las preguntas dirigidas a docentes y estudiantes, atendiendo a las diferencias propias de cada grupo.

4. El último paso fue, a partir de los ítems de esta matriz, elaborar los dos cuestionarios ad hoc en escala de tipo Likert con cinco opciones. Uno dirigido a los profesores que imparten docencia en estos entornos virtuales y otro destinado a los estudiantes para recabar su percepción de la incorporación de estos valores en los diseños instructivos (Rivas, 2014). 
Este cuestionario consta de 2 partes, una primera dirigida a la recogida de datos sociodemográficos de ambos grupos y su conocimiento e identificación de los valores propios de la misión y visión de esta universidad. La segunda se centró en su percepción sobre la incorporación y transmisión de estos valores en las asignaturas impartidas en los EVA. Este instrumento inicial fue validado por 8 expertos, seleccionados de acuerdo a los criterios de reconocida trayectoria investigadora en el área objeto de estudio, familiaridad con la educación a distancia y/o la formación en valores y, en lo posible, conocimiento de la institución en que se enmarca la presente investigación. En el protocolo de validación se les solicitó el grado de relevancia del contenido, claridad, y adecuación a los destinatarios de cada ítem en una escala del 1 (valor mínimo) y 5 (valor máximo) y la extensión del instrumento. Para la eliminación de un ítem del cuestionario se adoptó el criterio general de que el experto le asignara un valor menor a tres $(<3)$. El resultado de la validación de las preguntas de este instrumento se recoge en la Tabla 1.

\begin{tabular}{lcccc}
\hline \multirow{2}{*}{ RESUMEN } & \multicolumn{2}{c}{ PARTE A } & \multicolumn{2}{c}{ PARTE B } \\
\cline { 2 - 5 } & Profesores & Estudiantes & Profesores & Estudiantes \\
\hline Ítems modificados & 6 & 4 & 17 & 13 \\
\hline Ítems no modificados & 3 & 0 & 29 & 28 \\
\hline Ítems añadidos & 0 & 0 & 1 & 0 \\
\hline Ítems eliminados & 0 & 0 & 4 & 3 \\
\hline \multicolumn{1}{c}{ Ítems cuestionario } & 9 & 4 & 47 & 41 \\
\hline
\end{tabular}

Tabla 1. Resultados de la validación de las preguntas de los cuestionarios de profesores y estudiantes

Elaborado el cuestionario definitivo, se realizó la prueba piloto en línea con 48 docentes y 33 estudiantes. Este paso confirmó la consistencia interna de ambos protocolos — profesores y estudiantes_- mediante la prueba de fiabilidad Alfa de Cronbach, tanto a la totalidad de ambos (Tabla 2), como a cada uno de los valores que se evaluaban, lo que confirma su alto nivel de fiabilidad.

\begin{tabular}{cc}
\hline \multicolumn{2}{c}{ Alfa de Cronbach del cuestionario } \\
\hline Profesores-tutores & Estudiantes \\
\hline 0,933 & 0,966 \\
\hline
\end{tabular}

Tabla 2. Resultados prueba de fiabilidad en los cuestionarios de profesores y estudiantes

El análisis de datos se ha llevado a cabo mediante el programa SPSS (versión 20). En primer lugar se realizaron los análisis descriptivos de cada ítem. Seguidamente se procedió a efectuar los análisis estadísticos no paramétricos, aplicando la prueba de Kruskal Wallis, lo que nos permitió conocer si las respuestas, de las cuatro áreas de conocimiento que engloban las titulaciones que se imparten en UTPL, (Sociohumanística (S-H), Administrativa (Adm.), Técnica (Téc) y Biológica (Blg). eran 
estadísticamente similares. Para conocer entre qué áreas se daban diferencias, se utilizó el estadístico U de Mam Whitney.

Por último, reseñar los valores identificados en la competencia ‘trabajo en equipo', ya mencionados más arriba: cómo se entienden y el número de ítems que abordan este valor en cada uno de estos cuestionarios (Tabla 3).

\begin{tabular}{|c|c|c|c|c|}
\hline \multirow[b]{2}{*}{ Valor } & \multirow[b]{2}{*}{ Descripción } & \multirow[b]{2}{*}{ Indicador } & \multicolumn{2}{|c|}{$\mathbf{N}^{0}$ item } \\
\hline & & & Profesores & Estudiantes \\
\hline Confianza & $\begin{array}{l}\text { Generar un clima } \\
\text { de acogida y ayuda } \\
\text { hacia los demás. }\end{array}$ & Nivel de confianza & 53 y 54 & 41 \\
\hline Diálogo & $\begin{array}{l}\text { Actitud de acogida } \\
\text { hacia los demás y } \\
\text { capacidad de } \\
\text { intercambio de } \\
\text { ideas en un clima } \\
\text { de paz. }\end{array}$ & $\begin{array}{l}\text { Nivel de acogida de } \\
\text { las personas e } \\
\text { intercambio de ideas }\end{array}$ & $\begin{array}{c}38,39,40 \mathrm{y} \\
41\end{array}$ & 30,31 y 32 \\
\hline Responsabilidad & $\begin{array}{l}\text { Asumir con } \\
\text { puntualidad, } \\
\text { esmero, dedicación } \\
\text { los compromisos } \\
\text { adquiridos. }\end{array}$ & $\begin{array}{l}\text { Grado de logro de la } \\
\text { misión formativa y de } \\
\text { las responsabilidades } \\
\text { docentes }\end{array}$ & $\begin{array}{c}55,56,57,58, \\
59,60,61\end{array}$ & $\begin{array}{c}42,43,44,45, \\
46,47 \text { y } 48\end{array}$ \\
\hline $\begin{array}{l}\text { Generosidad / } \\
\text { Servicio-Solidaridad }\end{array}$ & $\begin{array}{l}\text { Disposición de } \\
\text { ayudar y compartir } \\
\text { los conocimientos y } \\
\text { la vida a favor de } \\
\text { los demás. } \\
\text { Dedicarle el tiempo } \\
\text { y apoyo que } \\
\text { necesitan. }\end{array}$ & $\begin{array}{l}\text { Grado de disposición } \\
\text { para ayudar a los } \\
\text { demás. }\end{array}$ & $\begin{array}{c}25,26,27,28, \\
29 \text { у } 30\end{array}$ & $\begin{array}{c}19,20,21, y \\
22\end{array}$ \\
\hline Respeto & $\begin{array}{l}\text { Atención a las } \\
\text { personas acorde a } \\
\text { su dignidad } \\
\text { humana. Atenderles } \\
\text { oportunamente con } \\
\text { la calidad y calidez } \\
\text { que merecen las } \\
\text { personas. }\end{array}$ & $\begin{array}{l}\text { Grado de buen trato y } \\
\text { atención a las } \\
\text { personas. }\end{array}$ & $\begin{array}{c}31,32,33,34, \\
35,36 \text { у } 37\end{array}$ & $\begin{array}{c}23,24,25,26, \\
27,28 \text { у } 29\end{array}$ \\
\hline
\end{tabular}

Tabla 3. Descripción de los valores implícitos en ‘Trabajo en equipo’ y los ítems con los que se han valorado en ambos cuestionarios

En cuanto a la fiabilidad de estos valores, se comprueba también el alto índice de fiabilidad de los ítems centrados en su valoración (Tabla 4): 


\begin{tabular}{ccc}
\hline \multicolumn{3}{c}{$\begin{array}{c}\text { Alfa de Cronbach de los valores vinculados a } \\
\text { 'Trabajo en equipo' }\end{array}$} \\
\hline & Profesores-tutores & Estudiantes \\
\hline Responsabilidad &, 93 &, 96 \\
\hline Diálogo &, 93 &, 96 \\
\hline Confianza &, 93 &, 96 \\
\hline Generosidad &, 93 &, 96 \\
\hline Respeto &, 93 &, 96 \\
\hline
\end{tabular}

Tabla 4. Resultados prueba de fiabilidad en los ítems relativos a los valores

relacionados con 'trabajo en equipo' en los cuestionarios de profesores y estudiantes

\section{Análisis y discusión de los resultados.}

La jerarquización de los valores implícitos en la misión de la universidad y en la educación a distancia

Para estimar el nivel de logro de nuestros estudiantes en los valores vinculados a la competencia del trabajo en equipo, debemos analizar aquellos ítems dirigidos a evaluar la confianza, el diálogo, la responsabilidad, la generosidad y el respeto en las propuestas formativas de las titulaciones de los EVA, tal como hemos expuesto anteriormente.

Con el fin de descubrir la jerarquía real de los valores institucionales más relevantes percibidos por profesores y estudiantes, se les solicitó que los priorizaran, contrastándolos, a su vez, con los valores propios de la educación a distancia (Tabla 5). Como resultado destacar que los valores relativos al trabajo en equipo no son los mejor considerados, figurando en la prioridad 1 únicamente dos de ellos entre los 5 primeros y tres en los últimos 5 puestos. Llama la atención que no se hayan destacado la solidaridad, la confianza o el diálogo y que ninguno supere el $50 \%$ como primera prioridad de ambos grupos, en especial entre los estudiantes, en los que se valora con un escaso $21 \%$. En suma, los valores que impulsan el trabajo en equipo no son los prioritarios, lo que nos indica que esta competencia no es una de las más trabajadas, o se favorezca en estos estudios, lo que se contrapone con las demandas de la sociedad. 


\begin{tabular}{|c|c|c|c|c|c|}
\hline \multicolumn{3}{|c|}{ Profesores } & & \multicolumn{2}{|l|}{ Estudiantes } \\
\hline \multirow{16}{*}{ 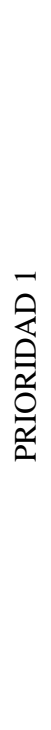 } & Valor & $\begin{array}{c}\% \\
\text { docentes } \\
\end{array}$ & \multirow{16}{*}{ 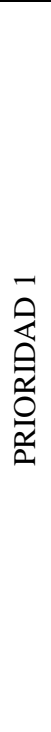 } & Valor & $\begin{array}{c}\% \\
\text { estudiantes } \\
\end{array}$ \\
\hline & 1. Amor a la verdad & $52,46 \%$ & & 1. Amor a la verdad & $33,92 \%$ \\
\hline & 2. Honestidad & $49,39 \%$ & & 2. Honestidad & $28,19 \%$ \\
\hline & 3. Respeto & $47,57 \%$ & & 3. Respeto & $21,92 \%$ \\
\hline & 4. Humildad & $42,72 \%$ & & 4. Responsabilidad & $18,39 \%$ \\
\hline & 5. Responsabilidad & $42,72 \%$ & & 5. Esfuerzo & $15,46 \%$ \\
\hline & 6. Libertad & $36,96 \%$ & & 6. Compromiso & $14,98 \%$ \\
\hline & 7. Compromiso & $35,15 \%$ & & 7. Disciplina & $13,45 \%$ \\
\hline & 8. Disciplina & $35,15 \%$ & & 8. Humildad & $13,27 \%$ \\
\hline & 9. Generosidad/ Solidaridad & $34,54 \%$ & & 9. Perseverancia & $12,60 \%$ \\
\hline & 10. Diálogo & $33,33 \%$ & & 10. Libertad & $11,51 \%$ \\
\hline & 11. Perseverancia & $33,03 \%$ & & 11. Generosidad/Solidaridad & $9,37 \%$ \\
\hline & 12. Tolerancia & $31,81 \%$ & & 12. Diálogo & $8,64 \%$ \\
\hline & 13. Confianza & $31,21 \%$ & & 13. Tolerancia & $5,90 \%$ \\
\hline & 14. Esfuerzo & $30,30 \%$ & & 14. Confianza & $5,78 \%$ \\
\hline & 15. Amabilidad & $27,57 \%$ & & 15. Amabilidad & $4,08 \%$ \\
\hline
\end{tabular}

Tabla 5. Jerarquización de valores como primera prioridad en la población de profesores y estudiantes

Dada la limitación lógica de espacio en este artículo, únicamente procedemos a analizar los resultados de 2 valores relativos a la competencia 'trabajo en equipo' situados en los primeros puestos de la jerarquía de profesores y estudiantes: respeto y responsabilidad.

\section{Respeto}

Sin duda, valor transversal a todas las competencias, ya que donde hay carencia de respeto, no puede haber trabajo en equipo, ni convivencia, ni trabajo eficaz y, por lo tanto, tampoco se puede dar diálogo, solidaridad, confianza, etc.

Hemos abordado este valor con siete ítems en ambas poblaciones, que analizan conductas concretas en los profesores y su reflejo en la formación de los estudiantes. Se hace referencia a ámbitos que tienen que ver con el cumplimiento de labores docentes en entornos virtuales, pues consideramos que cuando éstas se cumplen de manera apropiada, los estudiantes perciben que son respetados por sus profesores.

El sentir de la mayoría de los profesores (91,6\%) es que siempre y casi siempre toman en cuenta las opiniones de sus compañeros a pesar de que sean distintas a las propias. Esta percepción es similar en las cuatro áreas de conocimiento. En cambio, las puntuaciones de los estudiantes difieren en cuanto que su mayor porcentaje se encuentra en el rango algunas veces, siendo significativamente distinta la percepción entre las áreas S-H y Adm. Esto se evidencia especialmente en el rango más alto, en el 
cual el 23\% de estudiantes del área $\mathrm{S}-\mathrm{H}$ perciben que sus profesores respetan sus opiniones, aunque éstas sean distintas a las suyas. Mientras que en el área Adm. este rango alcanza el 14\%: no perciben ese respeto a sus ideas.

Una de las funciones de mayor relevancia en todo entorno virtual es la tutoría, en la que resulta crucial el respeto entre todos los participantes. Una concreción de este valor es la referencia a la puntualidad de los profesores al impartir las tutorías virtuales, resultado en el que hay diferencias significativas entre el área S-H, Adm. y Blg. En las cuatro áreas de conocimiento los porcentajes más altos de profesores se ubican en el rango siempre. Destaca el área Téc., en la cual el 77\% de los profesores manifiestan ser puntuales a la hora de impartirlas. Sin embargo, únicamente el 21\% de los estudiantes de esta misma área perciben que sus profesores siempre son puntuales en esta actividad. El mayor porcentaje de estudiantes, respecto a este ítem, tiende al rango central algunas veces.

Otro ítem hace referencia a la preparación de las tutorías virtuales por los profesores. Los porcentajes más altos se sitúan en el rango siempre, destacando, nuevamente, el área Téc. (83\%). Y las diferencias significativas se encuentran entre las áreas S-H y Adm. La percepción de los estudiantes en este punto muestra también diferencias significativas en las mismas áreas que en el caso de los profesores y, adicionalmente, entre las áreas S-H y Téc. En la población de estudiantes el porcentaje mayor se sitúa en casi siempre con valores similares (36\% y 37\%), excepto el área Téc. que alcanza el 31\%. Volvemos a llamar la atención sobre la significatividad de la diferencia de puntuaciones en ambos grupos.
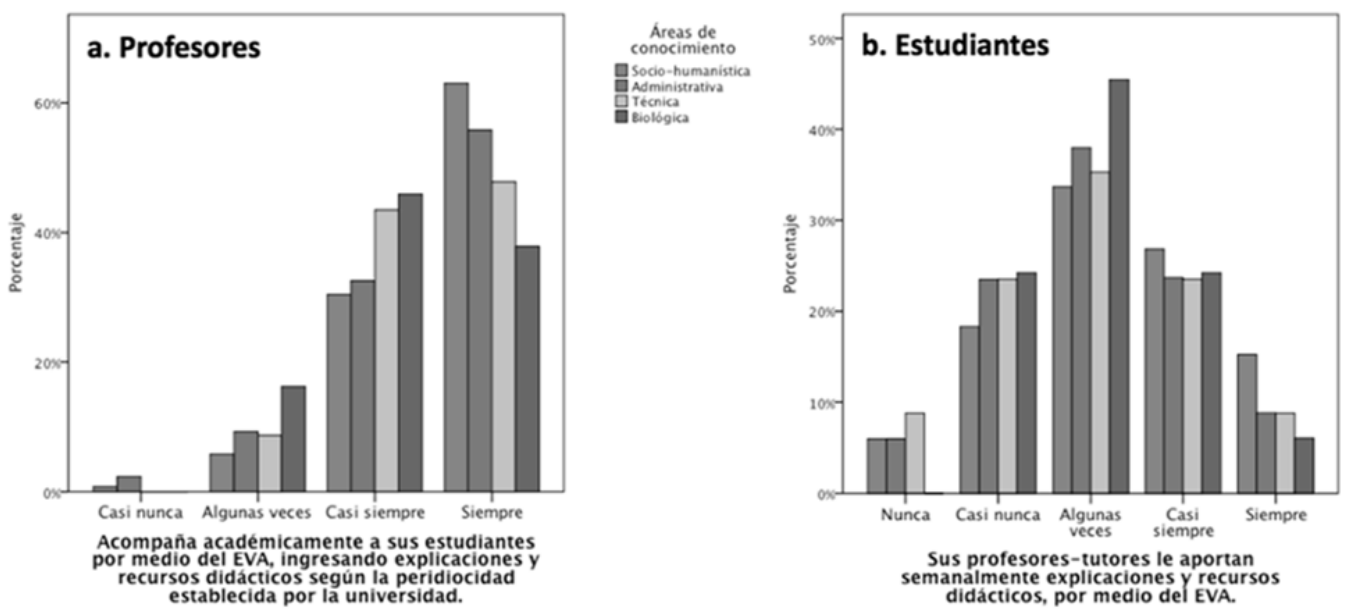

Figura 1. Comparación por áreas de conocimiento del ítem relativo al acompañamiento académico de los profesores a los estudiantes en el EVA. 
Se observa que hay una gran diferencia entre el sentir docente, respecto al acompañamiento a sus estudiantes por medio de este entorno y la percepción de estos. En el caso de los profesores hay diferencia significativa entre las áreas S-H y Blg. y, en el caso de los estudiantes, entre las áreas S-H y Adm. / S-H y Téc.

En la interacción asíncrona entre profesores y estudiantes por medio del correo electrónico, se observa una diferencia respecto a la percepción de los profesores. En este caso, el mayor porcentaje de profesores se sitúa en el rango casi siempre, a excepción del área S-H cuyo mayor porcentaje se ubica en siempre. Mientras se dan diferencias significativas en las respuestas de los estudiantes (Figura 2).
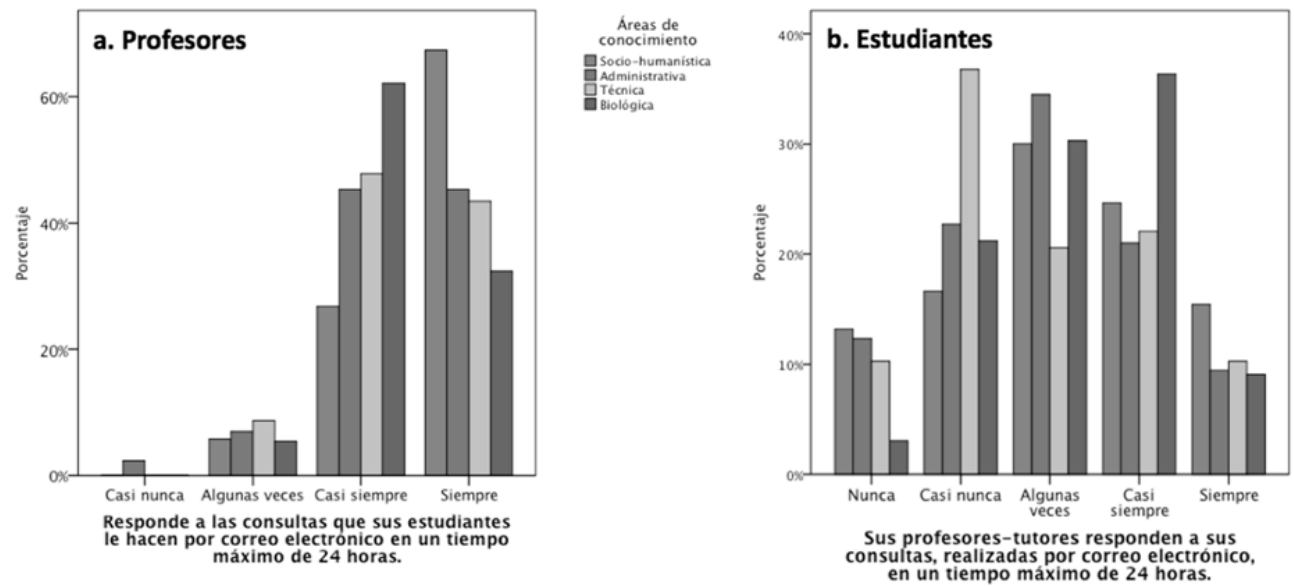

Figura 2. Comparación por áreas de conocimiento del ítem relativo a la atención a los estudiantes a través del correo electrónico.

En cuanto al ítem que evalúa el clima de respeto y empatía que los profesores fomentan en sus estudiantes, y cómo lo perciben estos últimos, podemos ver que en el caso de los profesores hay diferencias estadísticamente significativas entre las áreas SH y Adm., y la S-H y Blg., siendo los profesores del área S-H los que expresan un sentir superior en el rango siempre. En cuanto a la percepción de los estudiantes, la diferencia significativa está únicamente entre el área S-H y Adm. También en este caso el porcentaje mayor de estudiantes que se ubican en el rango siempre son los del área S-H (Figura 3). 

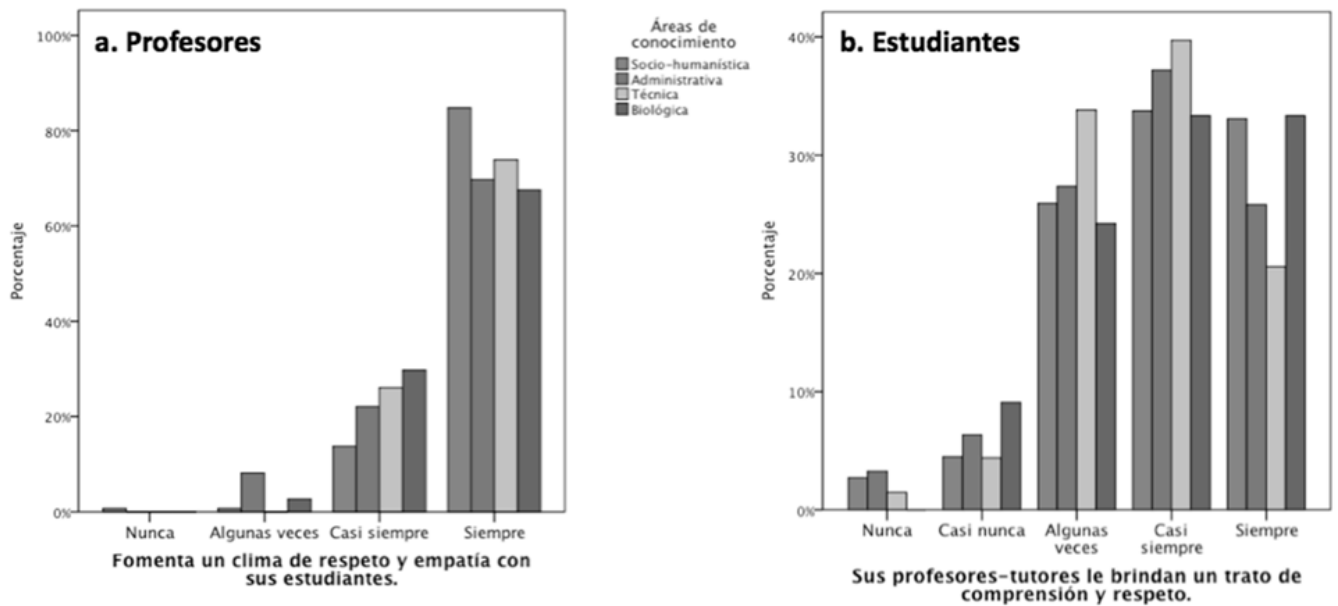

Figura 3. Comparación por áreas de conocimiento del ítem relativo al clima de respeto y empatía.

En el indicador referido a la capacidad de escucha y de aceptación de ideas y sugerencias de los estudiantes, la mayoría de los profesores se ubican en los dos rangos más altos, siempre y casi siempre, resultados que no muestran diferencias significativas entre las cuatro áreas de conocimiento. Sin embargo, la percepción de los estudiantes no tiene relación con estos resultados, destacando diferencias significativas entre el área S-H y Adm. (Figura 4).

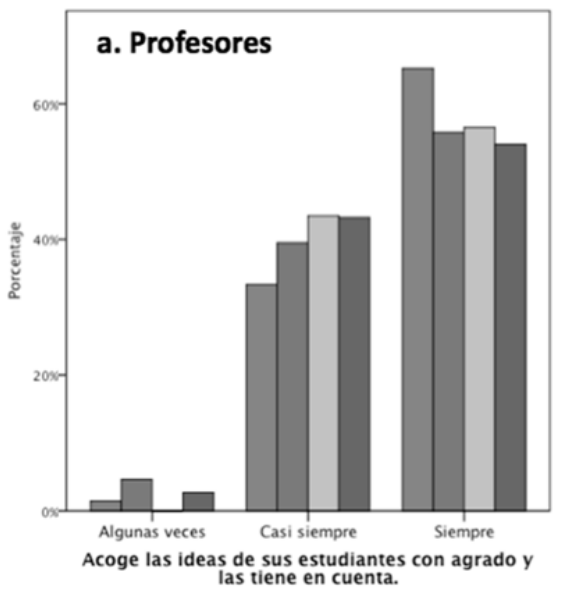


Figura 4. Comparación por áreas de conocimiento del ítem relativo a la capacidad de escucha y de aceptación de ideas y sugerencias de los estudiantes.

En este valor, si bien el porcentaje de profesores no siempre se concentra en el rango más alto al estar repartido entre siempre y casi siempre, la diferencia, en relación a la apreciación de los estudiantes, es importante. El hecho de que el mayor porcentaje de profesores se divida entre los dos rangos superiores, deja entrever que estos perciben, en alguna medida, que les falta mayor atención a los estudiantes en los aspectos evaluados y se confirma que hay deficiencias, en la atención a los estudiantes, relacionadas con la falta de cumplimiento de sus responsabilidades docentes o con la calidad de las mismas. Esta falta de tutela la sienten los estudiantes al identificar las principales carencias de la actuación docente, con la falta de acompañamiento de estos en el EVA y la respuesta a las consultas vía correo electrónico. Llama la atención que, cuando se les indicó a los profesores que señalaran los medios en los que se apoyaban para la transmisión de los valores (parte A del cuestionario), la mayoría manifestó que lo hacía a través del oportuno cumplimiento de sus responsabilidades docentes que, como podemos apreciar, no coincide con lo manifestado por los estudiantes. Y, en segundo lugar, indicaron que se apoyaban, prioritariamente, en el EVA que, justamente, es uno de los medios en que los estudiantes detectan una importante debilidad. En suma, la percepción de los estudiantes revela importantes debilidades docentes en la atención en el EVA, que deben ser analizadas para mejorar la actuación de nuestros profesores, además de trabajar con el alumnado lo que implica y exige cada uno de estos valores para ambos grupos.

\section{Responsabilidad}

Otro valor clave implícito en todo trabajo en equipo es de 'responsabilidad': cuando falta o esta se debilita, es difícil potenciar el trabajo colaborativo y, en ocasiones, se vuelve imposible, pues para que sea eficaz se requiere, que todos realicen las tareas asignadas correctamente y en el tiempo establecido. Resulta crucial el cumplimiento de las responsabilidades docentes respecto al estudiante, en cualquiera de las modalidades de estudio, más si cabe en educación a distancia, donde se requiere ese acompañamiento y cercanía de su profesor a lo largo de todo el proceso formativo (García Aretio, 2014).

Con el fin de identificar las variables que expresan mayores fortalezas y debilidades en el valor 'responsabilidad' para este análisis optamos por el rango más alto: siempre. El motivo de esta decisión se debe a que nuestro estudio es de carácter global, es decir, no hacemos referencia a profesores y estudiantes específicos, sino a toda la experiencia docente y discente del período elegido (Tabla 6). 


\begin{tabular}{|c|c|c|c|c|c|c|c|c|c|}
\hline \multicolumn{5}{|c|}{ Población de profesores } & \multicolumn{5}{|c|}{ Población de estudiantes } \\
\hline \multirow{3}{*}{$\begin{array}{c}\text { Ítems (7) del } \\
\text { valor } \\
\text { responsabilidad }\end{array}$} & \multicolumn{4}{|c|}{ Áreas de conocimiento } & \multirow{3}{*}{$\begin{array}{c}\text { Ítems (7) del } \\
\text { valor } \\
\text { responsabilidad }\end{array}$} & \multicolumn{4}{|c|}{ Áreas de conocimiento } \\
\hline & S-h. & A. & T. & B. & & S-h. & A. & T. & B. \\
\hline & $65 \%$ & $47 \%$ & $42 \%$ & $51 \%$ & & $31 \%$ & $23 \%$ & $22 \%$ & $26,5 \%$ \\
\hline
\end{tabular}

Tabla 6. Porcentajes del rango Siempre en profesores y estudiantes de las diferentes áreas de conocimiento en los 7 ítems del valor 'Responsabilidad'.

En el grupo de profesores destaca el área S-H (65\%), descendiendo el siguiente resultado a un 51\% situándose las otras dos áreas en un nivel inferior. Confrontando estos resultados de los profesores con la percepción de los estudiantes en estas mismas preguntas, podemos observar que hay diferencias significativas entre ambas poblaciones, pero existe una cierta correlación ya que, en el caso de los estudiantes, los mayores porcentajes, en el rango siempre, se dan en el área S-H (31\%), y en la Blg. $(26,5 \%)$. De todas formas, en ambos grupos estas puntuaciones resultan sumamente bajas.

Las tareas en las cuales los docentes sienten menor responsabilidad tienen que ver con la promoción de valores en las guías didácticas y la motivación para el trabajo autónomo. Esta percepción coincide en las cuatro áreas de conocimiento. Esto evidencia una debilidad axiológica por parte de los profesores, ya que la elaboración de las guías didácticas es un elemento clave en entornos virtuales, al ser uno de los medios fundamentales de interacción académica (Romero Fernández y Rubio Gómez, 2006; García Aretio, 2014). Otro aspecto en el que los profesores de todas las áreas, excepto en la Téc., manifiestan menor responsabilidad es la falta de atención a las dificultades de aprendizaje al elaborar estas guías didácticas. Por el contrario, el profesorado del área Téc. muestra mayor debilidad al interaccionar con sus estudiantes en los distintos canales de comunicación. Lo que nos lleva a proponer un estudio pormenorizado para detectar los problemas que están suscitando estas carencias en la atención a sus alumnos.

Cotejando estos datos con la percepción de los estudiantes, la mayor debilidad que detectan en sus profesores es que no reconocen en estas acciones dirigidas a una formación integral, es decir, lo contrario a lo que manifiestan los docentes en ese mismo ítem. También detectan debilidad, especialmente en el área S-H, en la ayuda de sus profesores por medio de una bibliografía complementaria que favorezca la consolidación de conocimientos. Esta última debilidad la perciben también los estudiantes del área Adm., además de no apreciar el apoyo de las guías didácticas para comprender, asimilar y profundizar en las respectivas materias. Este último aspecto también lo detectan los estudiantes del área Téc.

En el área Blg. la mayor debilidad que revelan los estudiantes, respecto a sus profesores, reside en la carencia de elementos motivadores en las guías didácticas que les facilite el estudio. Destacan los ítems más deficientes aquellos que se relacionan con la responsabilidad docente, dirigidos a la generación de un constante y efectivo 
diálogo a través de los canales de comunicación propios de esta metodología. Cuestión crucial en esta modalidad educativa.

Los estudiantes de las cuatro áreas de conocimiento perciben que la mayor fortaleza de sus profesores se concreta en respetar el horario que establecen para atenderles y en la selección de los textos básicos que los consideran actualizados y de alta calidad científica.

\section{Unas primeras conclusiones}

Tras este artículo, y la investigación que lo sustenta, hay varias cuestiones que queremos destacar y en las que consideramos necesario profundizar:

Reflexionar sobre las competencias genéricas y sobre cómo estamos incorporándolas en la docencia en las diferentes titulaciones que impartimos en nuestras universidades, ya que tan relevantes son los conocimientos específicos de una profesión, como las destrezas, actitudes y valores que se están demandando en los diferentes escenarios de la sociedad actual y en cada profesión en particular.

Evaluar la docencia que estamos impartiendo, no sólo en cuanto a conocimientos, sino también en todos los procesos, contenidos y escenarios en las que se lleva a cabo. Sin duda, la universidad debe ser consciente de la fuerza educadora de todos sus escenarios, canales y actores.

Avanzar de forma coherente en la formación en competencias genéricas y en valores acordes con la misión y visión de cada universidad. Punto al que usualmente no se le presta atención, pero que colaborará para lograr mejores resultados en la formación que se imparte, garantizando así la calidad de toda institución universitaria.

Un elemento clave en la formación son los valores, por lo que evaluarlos es responsabilidad de todo docente. Máxime si queremos alcanzar una educación de calidad. Los resultados de esta investigación nos llevan a evidenciar la diferencia significativa entre lo que los docentes consideran que están desarrollando y transmitiendo y lo que los estudiantes perciben. Cuestión que debe hacernos reflexionar y proponer nuevas investigaciones, e involucrar a todos los que intervienen en este proceso con el objeto de detectar cuáles son los puntos en los que se da esta disrupción y proponer acciones concretas de mejora.

Como ejemplo de esta realidad se han expuesto los resultados de 2 valores, prioritarios para ambas poblaciones, relativos a la competencia 'trabajo en equipo'. Y en ambos casos queda plasmado que los valores analizados nos describen importantes diferencias entre lo que manifiestan los profesores y la percepción que los estudiantes tienen de la formación recibida. Es necesario que los docentes reflexionen sobre su desempeño en el que, a pesar de sus percepciones, no están brindando una formación efectiva de los valores reseñados. Trabajar estos resultados con el profesorado y analizar la percepción de sus estudiantes sobre los diferentes ámbitos de su actuación didáctica y educativa promoverán, por un lado, una concienciación de la relevancia de 
sus decisiones y actuaciones y, por otro, la promoción de nuevas acciones dirigidas al logro de estos valores clave para todo profesional y ciudadano.

\section{Referencias bibliográficas}

ANECA (2007). El Profesional Flexible en la Sociedad del Conocimiento: Nuevas Exigencias en la Educación Superior en Europa. (REFLEX). Recuperado el 10 de octubre de 2011 en: http://www.aneca.es/estudios/docs/InformeejecutivoANECA_jornadasREFLEXV2 $0 . p d f$

BENEITONE, P., et al. (2007). Reflexiones y perspectivas de la Educación Superior en América Latina. Informe Final-Proyecto Tuning-América Latina 2004-2007. Bilbao: Universidad de Deusto. Recuperado el 6 de julio de 2014 en: http://tuning.unideusto.org/tuningal/index.php?option=com_docman\&task=down\& bid $=5 \underline{4}$

BENEITONE, P.; GONZÁLEZ, J., WAGENAAR, R. (Eds.) (2014). Meta-perfiles y perfiles. Una nueva aproximación para las titulaciones en América Latina. Bilbao: Deusto.

BENHAYON, M. (2011). Perfil de competencias transversales en educación superior. Tesis Doctoral. Madrid: UNED.

BENITEZ ZAVALA, A. (2009). La Educación en valores en el ámbito de la educación superior. REICE. Revista Iberoamericana sobre Calidad, Eficacia y Cambio en Educación, 7(2), 116-129. Recuperado el 15 de marzo de 2013 en: http://www.rinace.net/reice/numeros/arts/vol7num2/art6.pdf

BLANCO, A. (2009). Desarrollo y evaluación de competencias en educación superior. Madrid: Editorial Narcea.

CLEMENTE, J.S. y ESCRIBA, C. (2013). Análisis de la percepción de las competencias genéricas adquiridas en la universidad. Revista de Educación, 362, 535 - 561 .

CONFERENCIA REGIONAL DE LA EDUCACIÓN SUPERIOR EN AMÉRICA LATINA Y EL CARIBE (CRES). (2009). Declaraciones y plan de acción. Perfiles Educativos, 31(125), 90-108. Recuperado el 10 de octubre de 2013 en: http://www.scielo.org.mx/scielo.php?script=sci_arttext\&pid=S018526982009000300007\&lng=es\&tlng=es.

COROMINAS, E. (2001). Competencias genéricas en la formación universitaria. Revista de Educación, 325, 299-321.

COROMINAS, E., et al. (2006). Percepciones del profesorado ante la incorporación de las competencias genéricas. Revista de Educación, 341, pp. 301-336. Recuperado el 22 de febrero de 2009 en: http://web.udg.edu/pedagogia/images/resultats_recerca.pdf 
DUART, J.M. (2003). Educar en valores en entornos virtuales de aprendizaje: realidades y mitos. Barcelona: Fundación para la Universidad Oberta de Catalunya. Recuperado el 15 de marzo de 2014 en: http://www.uoc.edu/dt/20173/

ESTEBAN BARA, F. y MARTÍNEZ, M. (2012). ¿Son universidades todas las universidades? La universidad como comunidad ética. Bordón. Revista de Pedagogía, 64 (3), 77-92.

FRESÁN OROZCO, M. (2009). Repensando la idea de universidad en el siglo XXI. Una reflexión sobre el pensamiento de Pablo Carlevaro. Revista Reencuentro, 56, 52-59. Recuperado el 22 de febrero de 2009 en: http://www.redalyc.org/articulo.oa?id=34011860010>

GARCÍA ARETIO, L. (2009). Valores (en y de) la educación a distancia, en L.M. Romero (coord.) Construyendo espacios comunes de educación superior. I Congreso del Programa Campus de la Organización Universitaria interamericana (OUI). Loja, Ecuador. [documento policopiado]

GARCÍA ARETIO, L. (2014). Bases, mediaciones y futuro de la educación a distancia en la sociedad digital. Madrid: Síntesis.

GARCÍA LÓPEZ, R., et al. (2009). La formación ética profesional desde la perspectiva del profesorado universitario. Teoría de la Educación. Revista Interuniversitaria. 21(1), 199-221

GARCÍA MUÑOZ, T. (2003). El cuestionario como instrumento de investigación/evaluación. Recuperado el 22 de febrero de 2014 en: http://www.redalyc.org/articulo.oa?id=34011860010:http://cvonline.uaeh.edu.mx/C ursos/Maestria/MTE/Gen02/seminario_de_tesis/Unidad_4_anterior/Lect_El_Cuesti onario.pdf

GARCÍA-VALCARCEL, A.; HERNANDEZ MARTIN, A. y RECAMON, A. (2012). La metodología del aprendizaje colaborativo a través de las TIC: una aproximación a las opiniones de profesores y alumnos. Revista Complutense de Educación, 23(1), 161- 188.

HIRSCH, A. (2007). Ética profesional en estudiantes de posgrado. El caso de la Universidad Nacional Autónoma de México, en G. Chávez, A. Hirsch, y H. Maldonado (coords) México. Investigación en educación y valores, 143-155. México: Red Nacional de Investigadores en Educación y Valores/ Ediciones Gernika.

LÓPEZ VALVERDE, C. E. (2001). Educación a distancia y transmisión de valores. Revista Espiga. 3, 93-107.

MALDONADO, H. (2010). Lo esencial de los valores. Monterrey. México: Centro de Altos Estudios e Investigación Pedagógica. Recuperado el 15 de marzo de 2014 en: http://www.caeip.org/docs/altos-estudios/valores.pdf

MARTÍNEZ, M. (2006). Formación para la ciudadanía y educación superior. Revista Iberoamericana de Educación, 42, 85-102. 
MARTÍNEZ, M. (2008). Aprendizaje, servicio y responsabilidad social de las universidades. Barcelona: Octaedro/ ICE-UB.

MÉNDEZ MARTÍNEZ, J. (2003). Educación en valores y educación a distancia: una propuesta cognoscitiva. Revista Etic@Net.1, 1-6. Recuperado el 15 de marzo de 2011 en:

http://www.ugr.es/ sevimeco/UGR/revistaeticanet/Numero\%201/Articulos/Educen valores.pdf

MURILLO, A.C. (1994). Valores ético-sociales en la educación a distancia, en: Congreso Internacional sobre el problema de los valores en la educación a distancia. San José de Costa Rica: EUNED.

OECD (2005). The definition and selection of key competences. Executive summary. Paris: Organisation for Economic Co-operation and Development. Recuperado el 22 de febrero de 2014 en: http://www.oecd.org/dataoecd/47/61/35070367.pdf.

PÁEZ, H., ARREAZA, E. y VIZCAYA, W. (2008). Valores en la educación semipresencial. Revista Educación en Valores, 2 (10), 34-55. Recuperado el 15 de marzo de 2014 en: http://servicio.bc.uc.edu.ve/multidisciplinarias/educacion-envalores/v2n10/art5.pdf

PALLISERA, M. et al. (2010). La adaptación al EEES en España. Revista Iberoamericana de Educación. 52(4), 1 - 13.

PICHARDO, H. (2000). Valores que debe asumir, fomentar y defender la educación a distancia, en II Forum Iberoamericano de educación a distancia. La educación a distancia y los valores ante el siglo XXI. Antigua, Guatemala: Centro Iberoamericano de Formación de Antigua, Agencia Española de Cooperación Internacional (AECI). Recuperado el 15 de marzo de 2014 en: http://lanic.utexas.edu/project/laoap/cif/cif000028.pdf

REKALDE, I. y BUJAN, K. (2014). Las eRúbricas ante la evaluación de competencias transversales en educación superior. Revista Complutense de Educación, 25(2), 355-374.

RIVAS, R. (2014). La formación en valores en la educación superior a distancia. El caso de la Universidad Técnica Particular de Loja. Madrid, España: UNED. [Tesis Doctoral].

ROMERO FERNÁNDEZ, L. M.; RUBIO GÓMEZ, M. J. (2006). Universidad Técnica Particular de Loja, treinta años haciendo educación a distancia. Revista Iberoamericana de Educación a Distancia, 9 (1 y 2), 127-164.

RUBIO GÓMEZ, M. J (2000). Los valores de la educación en la esencia misma de la educación a distancia, en: II Forum Iberoamericano de educación a distancia. La educación a distancia y los valores ante el siglo XXI. Antigua, Guatemala: Centro Iberoamericano de Formación de Antigua, Agencia Española de Cooperación Internacional (AECI). 
RUIZ-CORBELLA, M. Y HARRIS, S. (coord.) (2012). La universidad como comunidad: recuperar la idea de universidad en el escenario del siglo XXI. Bordón. Revista de Pedagogía. 64(3), monográfico, 9 -148.

SÁNCHEZ-ELVIRA, M.A. et al. (2010). Análisis de las competencias genéricas en los nuevos títulos de Grado del EEES en las universidades españolas. REDU, 8(1), 35 73.

SÁNCHEZ-ELVIRA, M.A., et al. (2010). Del diseño de la evaluación en competencias genéricas: análisis empírico e intervención mediante rúbricas.

Programa de Estudios y Análisis. Proyecto EA2009-0102. Madrid: Ministerio de Educación.

SILAS, J., et al. (2007). El estudiante universitario frente a la pobreza, en G. Chávez, A. Hirsch, y H. Maldonado (coords) Investigación en educación y valores, 55-70. México: Red Nacional de Investigadores en Educación y Valores/ Ediciones Gernika.

TORRELLES, C. et al. (2011). Competencia de trabajo en equipo: definición y categorización. Profesorado, Revista de curriculum y formación del profesorado, 15(3), 329 - 344. Recuperado el 10 de diciembre de 2013 en: http://www.ugr.es/ recfpro/rev153COL8.pdf

TÜNNERMANN BERNHEIM, C. (2010). Las conferencias regionales y mundiales sobre educación superior de la UNESCO y su impacto en la educación superior de América Latina. Universidades, LX (Septiembre-Diciembre). Recuperado el 10 de diciembre de 2012 en: http://www.redalyc.org/articulo.oa?id=37318570005> ISSN 0041-8935

UNIVERSITY OF CAMBRIDGE (2008). Cambridge Undergraduate Transferable Skills Website: an interactive guide. Cambridge University. Recuperado el 22 de febrero de 2009 en: http://www.caret.cam.ac.uk/transkills/

VILLA, A. y POBLETE, M. (2008). Aprendizaje basado en competencias. Una propuesta para la evaluación de las competencias genéricas. Bilbao: Ediciones Mensajero.

VILLA, A. y POBLETE, M. (2011). Evaluación de competencias genéricas: principios, oportunidades y limitaciones. Bordón. Revista de Pedagogía, 63(1), 147 -170 .

YAÑIZ, C. y VILLARDON, L. (2012). Modalidades de evaluación de competencias genéricas en la formación universitaria. DIDAC. Revista Electrónica, 60, 15 - 19. 


\section{Correspondencia con las autoras}

Marta RUIZ-CORBELLA

Dpto Teoría de la Educación y Pedagogía Social

Facultad de Educación

Universidad Nacional de Educación a Distancia (UNED)

c/ Juan del Rosal, 14

28040 Madrid (España)

e-mail: mruiz@edu.uned.es

Rosario de RIVAS MANZANO

Modalidad Abierta y a Distancia

Universidad Técnica Particular de Loja - UTPL

c/ San Cayetano Alto, s/n

Loja (Ecuador)

e-mail: rrivas@utpl.edu.ec 